PROCEEDINGS OF THE

AMERICAN MATHEMATICAL SOCIETY

Volume 129, Number 4, Pages 1219-1228

S 0002-9939(00)05598-2

Article electronically published on September 25, 2000

\title{
A RESUlT ABOUt A SELECTION PROBLEM OF MICHAEL
}

\author{
FRANCIS JORDAN AND SAM B. NADLER, JR.
}

(Communicated by Alan Dow)

\begin{abstract}
It is shown that a continuum that is an $S_{4}$ space in the sense of Michael must be hereditarily decomposable. This improves known results, thereby providing more evidence that such continua must be dendrites.
\end{abstract}

\section{INTRODUCTION}

We let $2^{X}$ denote the space of all non-empty, compact subsets of a metric space $X ; 2^{X}$ has the Vietoris topology or, equivalently, the Hausdorff metric topology [7].

Let $\mathcal{F} \subseteq 2^{X}$. A continuous selection for $\mathcal{F}$ is a continuous function $\sigma: \mathcal{F} \rightarrow X$ such that $\sigma(F) \in F$ for each $F \in \mathcal{F}$.

Michael [6 p.178] defined an $S_{4}$ space to be a space $X$ such that there is a continuous selection for every $\mathcal{F} \subseteq 2^{X}$ such that the members of $\mathcal{F}$ are mutually disjoint and $\bigcup \mathcal{F}=X$. The question of which spaces are $S_{4}$ spaces is asked in [6] p.155], and some partial answers are in [6, pp.178-179].

It is known that $S_{4}$ spaces cannot contain a nondegenerate hereditarily indecomposable continuum (5.14 of [7, p.260]). We show that a nondegenerate, separable metric space that is an $S_{4}$ space can be separated by a countable set (Theorem 7.1 in section 7 which is somewhat stronger). Thus, a nondegenerate continuum that is an $S_{4}$ space must be hereditarily decomposable.

In the proof of Theorem 7.1 we use an idea that is new to the theory of continuous selections; namely, we use connectivity functions. Our results about connectivity functions seem to be of independent interest. In particular, we prove some results about connectivity bijections.

\section{Terminology}

We denote the real numbers by $\mathbb{R}$. The cardinality $\mathbb{R}$ is denoted by $\mathfrak{c}$. We denote the unit circle by $S^{1}$.

A continuum is a non-empty compact connected metric space.

If $X$ is a topological space and $A \subseteq X$ is such that $X \backslash A$ is separated (i.e., not connected), we say $A$ is a separator of $X$.

The 2 -fold symmetric product of $X$ with the Vietoris topology is denoted by $F_{2}(X)$, as in 7 .

Received by the editors July 9, 1998 and, in revised form, June 15, 1999.

1991 Mathematics Subject Classification. Primary 54C65, 54E40; Secondary 54F15.

Key words and phrases. Connectivity functions, continuous selections, $\mathfrak{c}$-connected, hereditarily decomposable continua, indecomposable continua, $S_{4}$ spaces.

(C)2000 American Mathematical Society 
We say that a space $X$ is $\mathfrak{c}$-connected provided that $X$ has no separator of cardinality less than continuum.

We say $X$ is weak $S_{4}$ space provided that there is a continuous selection for every $\mathcal{F} \subseteq F_{2}(X)$ such that the members of $\mathcal{F}$ are mutually disjoint and $\bigcup \mathcal{F}=X$. Clearly, every $S_{4}$ space is a weak $S_{4}$ space.

We use cl to denote closure.

\section{3. $S_{4}$ SPACES AND WEAK $S_{4}$ SPACES}

In [6, p.178] it is stated that $S^{1}$ is not an $S_{4}$ space and that finite trees are $S_{4}$ spaces. It is in fact easy to check that there is no continuous selection for $\mathcal{E}=\left\{\{x,-x\}: x \in S^{1}\right\} \subseteq 2^{S^{1}}$, where $-x$ denotes the point antipodal to $x$ in $S^{1}$.

We verify two basic facts about $S_{4}$ and weak $S_{4}$ spaces.

Proposition 3.1. Both properties $S_{4}$ and weak $S_{4}$ are hereditary with respect to subsets.

Proof. Suppose $X$ is an $S_{4}$ space and $A \subseteq X$. Let $\mathcal{E}$ be a cover of $A$ by mutually disjoint non-empty compact sets. To find a continuous selection for $\mathcal{E}$ consider the cover of $X$ by mutually disjoint non-empty compact sets given by $\mathcal{E}^{*}=\mathcal{E} \cup\{\{x\}: x \in$ $X \backslash A\}$. Since $X$ is $S_{4}$, there is a continuous selection $\Psi: \mathcal{E}^{*} \rightarrow X$. The restriction $\Psi \mid \mathcal{E}: \mathcal{E} \rightarrow A$ is a continuous selection for $\mathcal{E}$. Thus, $A$ is an $S_{4}$ space. The argument for weak $S_{4}$ spaces is similar.

Proposition 3.1 implies that if a space $X$ can be homeomorphically embedded into an $S_{4}$ or weak $S_{4}$ space, then $X$ is $S_{4}$ or weak $S_{4}$. In fact, we can say a little bit more:

Proposition 3.2. Suppose $Y$ is an $S_{4}$ (weak $S_{4}$ ) space and $f: X \rightarrow Y$ is continuous and injective. Then $X$ is an $S_{4}$ (weak $\left.S_{4}\right)$ space.

Proof. Assume $Y$ is an $S_{4}$ space. Let $F: 2^{X} \rightarrow 2^{Y}$ be the map induced by $f$, that is, $F(A)=f[A]$ for each $A \in 2^{X}$. Note that $F$ is continuous. Let $\mathcal{E}$ be a cover of $X$ by mutually disjoint non-empty compact sets. Since $f$ is injective and continuous, $\mathcal{E}^{*}=\{f[E]: E \in \mathcal{E}\}$ is a cover of $f[X]$ by mutually disjoint non-empty compact sets. By Proposition $3.1 f[X] \subseteq Y$ is an $S_{4}$ space. So, there is a continuous selector $\Psi^{*}: \mathcal{E}^{*} \rightarrow f[X]$. Define $\Psi: \mathcal{E} \rightarrow X$ by $\Psi(E)=\left(f^{-1} \circ \Psi^{*} \circ F\right)(E)$. Clearly, $\Psi$ is a selection. We show $\Psi$ is continuous. Let $E_{n} \in \mathcal{E}$ and $\lim _{n \rightarrow \infty} E_{n}=E$. We show that $\lim _{n \rightarrow \infty} \Psi\left(E_{n}\right)=\Psi(E)$. Let $\mathcal{K}=\left\{E_{n}: 1 \leq n<\infty\right\} \cup\{E\}$ and let $K=\bigcup \mathcal{K}$. Notice that

$$
\left(\Psi^{*} \circ F\right)[\mathcal{K}]=\Psi^{*}\left[\{f[E]\} \cup\left\{\left\{f\left[E_{n}\right]\right\}: 1 \leq n<\infty\right\}\right] \subseteq f[K] .
$$

It follows that $\Psi\left|\mathcal{K}=\left(f^{-1} \mid f[K] \circ \Psi^{*} \circ F\right)\right| \mathcal{K}$. Since $K$ is compact (by 5.6 of $[\underline{6}$, p. 168]), $f \mid K$ is a homeomorphism. Since $f \mid K$ is a homeomorphism, $f^{-1} \mid f[K]$ is continuous. Thus,

$\Psi\left|\left(\{E\} \cup\left\{\left\{E_{n}\right\}: 1 \leq n<\infty\right\}\right)=\left(f^{-1} \mid f[K] \circ \Psi^{*} \circ F\right)\right|\left(\{E\} \cup\left\{\left\{E_{n}\right\}: 1 \leq n<\infty\right\}\right)$

is continuous. Therefore, $\lim _{n \rightarrow \infty} \Psi\left(E_{n}\right)=\Psi(E)$.

The proof for the case when $X$ is a weak $S_{4}$ space is similar. 


\section{Connected Spaces}

We prove some useful facts about $\mathfrak{c}$-connected spaces and connected spaces in general. The following result may be known, but we include a proof for completeness.

Lemma 4.1. Let $X$ be a metric space, and let $D \subseteq X$. Then $D \subseteq X$ is a dense connected subspace of $X$ if and only if $D$ has non-empty intersection with every closed separator of $X$.

Proof. Suppose $D$ is a dense connected subset of $X$. By way of contradiction, assume there is a closed separator $F$ of $X$ such that $F \cap D=\emptyset$. Let $X \backslash F=U \cup V$ where $U$ and $V$ are disjoint non-empty open subsets of $X$. Since $D$ is connected, $D \cap U=\emptyset$ or $D \cap V=\emptyset$; in either case the density of $D$ is violated. So $F \cap D \neq \emptyset$.

Suppose $D$ has non-empty intersection with every closed separator of $X$. We show $D$ is connected. If $D$ were separated, then $X \backslash D$ would be a separator of $X$. By [5, Theorem 3, p. 155], $X \backslash D$ contains a closed separator of $X$, but such a separator would have empty intersection with $D$ contradicting our assumptions about $D$. Thus, $D$ is connected. To see that $D$ is dense it is enough to notice that for any $x \in X$ and sufficiently small open neighborhoods $U$ of $x$, the boundary of $U$ is a closed separator of $X$.

Lemma 4.2. Let $Y$ be a c-connected nondegenerate separable metric space. Then there exist $\mathbf{c}$-connected dense sets $Y_{1}, Y_{2} \subseteq Y$ such that $Y=Y_{1} \cup Y_{2}$ and $Y_{1} \cap Y_{2}=\emptyset$.

Proof. Recall that separable metric spaces have at most $\mathfrak{c}$-many closed subsets. Let $\left\{T_{\alpha}\right\}_{\alpha \in \mathfrak{c}}$ be an enumeration of the closed separators of $Y$ such that each closed separator appears $\mathfrak{c}$-many times in the enumeration. We construct two sequences $\left\{a_{\alpha}\right\}_{\alpha \in \mathfrak{c}}$ and $\left\{b_{\alpha}\right\}_{\alpha \in \mathfrak{c}}$ of points in $Y$ such that for each $\alpha \in \mathfrak{c}$ we have

(1) $a_{\alpha}, b_{\alpha} \in T_{\alpha}$,

(2) $a_{\alpha} \notin\left\{a_{\beta}: \beta<\alpha\right\} \cup\left\{b_{\beta}: \beta \leq \alpha\right\}$, and

(3) $b_{\alpha} \notin\left\{b_{\beta}: \beta<\alpha\right\} \cup\left\{a_{\beta}: \beta \leq \alpha\right\}$.

Suppose $\alpha<\mathfrak{c}$ and we have constructed $\left\{a_{\beta}: \beta<\alpha\right\}$ and $\left\{b_{\beta}: \beta<\alpha\right\}$ satisfying (1), (2), and (3). We show how to pick $a_{\alpha}$ and $b_{\alpha}$ so that (1), (2), and (3) are satisfied. Since $T_{\alpha}$ separates $Y$, we have $\left|T_{\alpha}\right|=\mathfrak{c}$. Pick distinct points $a_{\alpha}, b_{\alpha} \in$ $T_{\alpha} \backslash\left(\left\{a_{\beta}: \beta<\alpha\right\} \cup\left\{b_{\beta}: \beta<\alpha\right\}\right)$. Clearly, $a_{\alpha}$ and $b_{\alpha}$ are as desired.

Let $Y_{1}=\left\{a_{\alpha}: \alpha<\mathfrak{c}\right\}$ and $Y_{2}=Y \backslash Y_{1}$. Clearly, $Y=Y_{1} \cup Y_{2}$ and $Y_{1} \cap Y_{2}=\emptyset$, and by (2) and (3) we have $\left\{b_{\alpha}: \alpha<\mathfrak{c}\right\} \subseteq Y_{2}$. Let $i \in\{1,2\}$. Suppose $S \subseteq Y_{i}$ and $|S|<\mathfrak{c}$. By (1) and the fact that each closed separator appears $\mathfrak{c}$-many times in the enumeration, $\left|T \cap Y_{i}\right|=\mathfrak{c}$ for every closed separator $T$ of $Y$. So, $T \cap\left(Y_{i} \backslash S\right) \neq \emptyset$ for any closed separator $T$ of $Y$. By Lemma 4.1 we have that $Y_{i} \backslash S$ is connected and dense in $Y$. Thus, $Y_{i}$ is dense in $Y$ and $\mathfrak{c}$-connected.

\section{Type 1 AND TYPE 2 SePARATORS}

To prove Theorem 7.1 we will need to notice that there are two basic types of closed separators of the product of two spaces:

Lemma 5.1. Let $X$ and $Y$ be connected spaces, let $S$ be a closed separator of $X \times Y$, and let

$$
H_{S}=\left\{x \in X: \pi_{X}^{-1}(x) \subseteq S\right\}
$$


Then one of the following holds:

(1) $H_{S}$ is a separator of $X$;

(2) there is a non-empty open set $U$ in $X$ such that $\pi_{X}^{-1}(x) \cap S$ is a separator of $\{x\} \times Y$ for all $x \in U$.

Proof. Let $(X \times Y) \backslash S=W \cup V$ where $W \cap V=\emptyset$ and both $W$ and $V$ are non-empty and open in $X \times Y$. Let $W_{1}=\pi_{X}(W)$ and $V_{1}=\pi_{X}(V)$. There two possible cases.

Case 1: $W_{1} \cap V_{1}=\emptyset$. We show that (1) holds. Notice that $X \backslash\left(W_{1} \cup V_{1}\right)$ separates $X$. Clearly, $H_{S}=X \backslash\left(W_{1} \cup V_{1}\right)$. So, $H_{S}$ is a separator of $X$.

Case 2: $W_{1} \cap V_{1} \neq \emptyset$. We show that (2) holds with $W_{1} \cap V_{1}=U$. Let $x \in U$. Since $(\{x\} \times Y) \cap W$ and $(\{x\} \times Y) \cap V$ are non-empty disjoint open subsets of $\{x\} \times Y$, it follows that $(\{x\} \times Y) \cap S$ separates $\{x\} \times Y$. So, $\pi_{X}^{-1}(x) \cap S$ is a separator of $\{x\} \times Y$. Thus, (2) holds with $U=W_{1} \cap V_{1}$.

For $i \in\{1,2\}$, we say that a closed separator $S$ of $X \times Y$ is a type $i$ separator in $X \times Y$ if $S$ satisfies (i) of Lemma 5.1 Note that a type $i$ separator of $X \times Y$ may not be a type $i$ separator of $Y \times X$, where $i \in\{1,2\}$.

Notice that the proof of Lemma 5.1 works for connected spaces in general.

\section{Connectivity Functions}

Throughout the remainder of this paper do not distinguish between a function and its graph.

A function $f: X \rightarrow Y$ is called a connectivity function provided that the graph of $f \mid C$ is connected for every connected subset $C$ of $X$. Connectivity functions have been studied extensively (e.g., [1]-[3]). We prove some results about connectivity functions and functions with connected graphs.

Lemma 6.1. Let $X, Y_{1}$, and $Y_{2}$ be nondegenerate connected separable metric spaces such that $Y_{1}$ and $Y_{2}$ are $\mathfrak{c}$-connected. Let $\mathcal{C}$ be a collection of $\leq \mathfrak{c}$ nondegenerate connected subsets of $X$. Then there is a function $F: X \rightarrow Y_{1} \times Y_{2}$ such that

(i) $\left|\left\{x \in X: y_{i}=\pi_{Y_{i}}(F(x))\right\}\right|=1$ for all $y_{i} \in Y_{i}$ and $i=1,2$;

(ii) $F \mid C$ is connected and dense in $C \times\left(Y_{1} \times Y_{2}\right)$ for each $C \in \mathcal{C}$.

Proof. Let $\left\{T_{\alpha}\right\}_{\alpha \in \mathfrak{c}}$ be an enumeration of all subsets of $X \times\left(Y_{1} \times Y_{2}\right)$ each of which is a type 2 (closed) separator of $C \times\left(Y_{1} \times Y_{2}\right)$ for some $C \in \mathcal{C}$. (Indeed, there are at most $\mathfrak{c}$ such sets $T_{\alpha}$ since $|\mathcal{C}| \leq \mathfrak{c}$ and $C \times\left(Y_{1} \times Y_{2}\right)$ has only $\mathfrak{c}$ closed sets for each $C \in \mathcal{C}$.)

We first show how to define six injective sequences

$$
\begin{gathered}
R_{1}=\left\{x_{\alpha} \in X\right\}_{\alpha \in \mathfrak{c}}, R_{2}=\left\{z_{\alpha} \in X\right\}_{\alpha \in \mathfrak{r}}, R_{3}=\left\{y_{\alpha}^{1} \in Y_{1}\right\}_{\alpha \in \mathfrak{c}}, R_{4}=\left\{y_{\alpha}^{2} \in Y_{2}\right\}_{\alpha \in \mathfrak{c}}, \\
R_{5}=\left\{w_{\alpha}^{1} \in Y_{1}\right\}_{\alpha \in \mathfrak{c}}, R_{6}=\left\{w_{\alpha}^{2} \in Y_{2}\right\}_{\alpha \in \mathfrak{c}}
\end{gathered}
$$

satisfying the following three conditions for all $\alpha \in \mathfrak{c}$ :

(1) $\left(x_{\alpha}, y_{\alpha}^{1}, y_{\alpha}^{2}\right) \in T_{\alpha}$;

(2) $x_{\beta} \neq z_{\lambda}$ for all $\beta, \lambda \leq \alpha$;

(3) $y_{\beta}^{i} \neq w_{\lambda}^{i}$ for all $\beta, \lambda \leq \alpha$ and $i=1$ and 2 .

We use transfinite induction, as follows: Assume that we have defined the terms of the sequences for all $\beta<\alpha$.

(a) Define $z_{\alpha}, w_{\alpha}^{1}$, and $w_{\alpha}^{2}$ as follows: Pick

$$
z_{\alpha} \in X \backslash\left\{x_{\beta}, z_{\beta} \in X: \beta<\alpha\right\}
$$


and, for $i=1$ and 2 , pick

$$
w_{\alpha}^{i} \in Y_{i} \backslash\left\{y_{\beta}^{i}, w_{\beta}^{i} \in Y_{i}: \beta<\alpha\right\} .
$$

(b) Define $x_{\alpha}$ as follows: Since $T_{\alpha}$ is a type 2 separator of $C \times\left(Y_{1} \times Y_{2}\right)$ for some $C \in \mathcal{C}$, there is a nonempty open set $U$ in $C$ such that $\pi_{X}^{-1}(x) \cap T_{\alpha}$ is a separator of $\{x\} \times\left(Y_{1} \times Y_{2}\right)$ for each $x \in U$. Now, noting that $|U|=\mathfrak{c}$, pick

$$
x_{\alpha} \in U \backslash\left\{x_{\beta}, z_{\gamma} \in X: \beta<\alpha \text { and } \gamma \leq \alpha\right\} .
$$

(c) Finally, define $y_{\alpha}^{1}$ and $y_{\alpha}^{2}$ as follows: Let $T_{\alpha}, U$, and $x_{\alpha}$ be as in (b), and let

$$
Q=\pi_{Y_{1} \times Y_{2}}\left[\pi_{X}^{-1}\left(x_{\alpha}\right) \cap T_{\alpha}\right] .
$$

Since $x_{\alpha} \in U, \pi_{X}^{-1}\left(x_{\alpha}\right) \cap T_{\alpha}$ is a closed separator of $\left\{x_{\alpha}\right\} \times\left(Y_{1} \times Y_{2}\right)$; hence, $Q$ is a closed separator of $Y_{1} \times Y_{2}$. We pick $y_{\alpha}^{1}$ and $y_{\alpha}^{2}$ according to two cases (which exhaust all possibilities by Lemma 5.1):

Case 1: $Q$ is a type 1 separator of $Y_{1} \times Y_{2}$. Then,

$$
H_{Q}=\left\{y \in Y_{1}: \pi_{Y_{1}}^{-1}(y) \subseteq Q\right\} \text { is a separator of } Y_{1} .
$$

Thus, since $Y_{1}$ is $\mathfrak{c}$-connected, $\left|H_{Q}\right|=\mathfrak{c}$. Hence, we can pick

$$
y_{\alpha}^{1} \in H_{Q} \backslash\left\{y_{\beta}^{1}, w_{\gamma}^{1} \in Y_{1}: \beta<\alpha \text { and } \gamma \leq \alpha\right\} .
$$

Pick

$$
y_{\alpha}^{2} \in Y_{2} \backslash\left\{y_{\beta}^{2}, w_{\gamma}^{2} \in Y_{2}: \beta<\alpha \text { and } \gamma \leq \alpha\right\} .
$$

Case 2: $Q$ is a type 2 separator of $Y_{1} \times Y_{2}$. Then there is a nonempty open set $V$ in $Y_{1}$ such that $\pi_{Y_{1}}^{-1}(y) \cap Q$ is a separator of $\{y\} \times Y_{2}$ for each $y \in V$. Thus, since $|V|=\mathfrak{c}$, we can pick

$$
y_{\alpha}^{1} \in V \backslash\left\{y_{\beta}^{1}, w_{\gamma}^{1} \in Y_{1}: \beta<\alpha \text { and } \gamma \leq \alpha\right\} .
$$

Since $Y_{2}$ is $\mathfrak{c}$-connected and $y_{\alpha}^{1} \in V,\left|\pi_{Y_{1}}^{-1}\left(y_{\alpha}^{1}\right) \cap Q\right|=\mathfrak{c}$; hence, we can pick

$$
y_{\alpha}^{2} \in \pi_{Y_{2}}\left[\pi_{Y_{1}}^{-1}\left(y_{\alpha}^{1}\right) \cap Q\right] \backslash\left\{y_{\beta}^{2}, w_{\gamma}^{2} \in Y_{2}: \beta<\alpha \text { and } \gamma \leq \alpha\right\} .
$$

We have finished defining the six injective sequences

$$
\begin{gathered}
R_{1}=\left\{x_{\alpha} \in X\right\}_{\alpha \in \mathfrak{c}}, R_{2}=\left\{z_{\alpha} \in X\right\}_{\alpha \in \mathfrak{c}}, R_{3}=\left\{y_{\alpha}^{1} \in Y_{1}\right\}_{\alpha \in \mathfrak{c}}, R_{4}=\left\{y_{\alpha}^{2} \in Y_{2}\right\}_{\alpha \in \mathfrak{c}}, \\
R_{5}=\left\{w_{\alpha}^{1} \in Y_{1}\right\}_{\alpha \in \mathfrak{c}}, R_{6}=\left\{w_{\alpha}^{2} \in Y_{2}\right\}_{\alpha \in \mathfrak{c} .}
\end{gathered}
$$

We show that the sequences satisfy $(1):\left(x_{\alpha}, y_{\alpha}^{1}, y_{\alpha}^{2}\right) \in T_{\alpha}$ for all $\alpha \in \mathfrak{c}$. In Case 1 we picked $y_{\alpha}^{1} \in H_{Q}=\left\{y \in Y_{1}: \pi_{Y_{1}}^{-1}(y) \subseteq Q\right\}$ and we picked $y_{\alpha}^{2} \in Y_{2}$. Hence, $\left(y_{\alpha}^{1}, y_{\alpha}^{2}\right) \in Q$. Therefore, since

$$
Q=\pi_{Y_{1} \times Y_{2}}\left[\pi_{X}^{-1}\left(x_{\alpha}\right) \cap T_{\alpha}\right],
$$

we have that $\left(x_{\alpha}, y_{\alpha}^{1}, y_{\alpha}^{2}\right) \in T_{\alpha}$. In Case 2, we picked $y_{\alpha}^{1} \in V \subseteq Y_{1}$ and we picked $y_{\alpha}^{2} \in \pi_{Y_{2}}\left[\pi_{Y_{1}}^{-1}\left(y_{\alpha}^{1}\right) \cap Q\right]$. Hence, $\left(y_{\alpha}^{1}, y_{\alpha}^{2}\right) \in Q$. Therefore, again we have that $\left(x_{\alpha}, y_{\alpha}^{1}, y_{\alpha}^{2}\right) \in T_{\alpha}$. This completes the proof of (1).

It is clear that the sequences $R_{1}, \ldots, R_{6}$ satisfy conditions (2) and (3).

Next, we define the function $F: X \rightarrow Y_{1} \times Y_{2}$. Note that $\left|R_{i}\right|=\mathfrak{c}$ for each $i=1, \ldots, 6$. Also, note that

$$
X \backslash R_{1} \supset R_{2}, Y_{1} \backslash R_{3} \supset R_{5}, Y_{2} \backslash R_{4} \supset R_{6} .
$$


Hence, there are bijections $g: X \backslash R_{1} \rightarrow Y_{1} \backslash R_{3}$ and $h: X \backslash R_{1} \rightarrow Y_{2} \backslash R_{4}$. Let

$$
F(x)= \begin{cases}\left(y_{\alpha}^{1}, y_{\alpha}^{2}\right), & x=x_{\alpha} \in R_{1}, \\ (g(x), h(x)), & x \in X \backslash R_{1} .\end{cases}
$$

Finally, we show that $F$ satisfies conditions (i) and (ii) of our lemma.

We prove (i) for $y_{1} \in Y_{1}$ (the proof for $y_{2} \in Y_{2}$ is similar). Fix $y_{1} \in Y_{1}$, and let

$$
n=\left|\left\{x \in X: y_{1}=\pi_{Y_{1}}(F(x))\right\}\right| .
$$

We first show that $n \geq 1$. If $y_{1} \in R_{3}$, say $y_{1}=y_{\alpha}^{1}$, then $F\left(x_{\alpha}\right)=\left(y_{\alpha}^{1}, y_{\alpha}^{2}\right)$ and, hence,

$$
\pi_{Y_{1}}\left(F\left(x_{\alpha}\right)\right)=y_{\alpha}^{1}=y_{1} .
$$

If $y_{1} \in Y_{1} \backslash R_{3}$, then, since $g\left(X \backslash R_{1}\right)=Y_{1} \backslash R_{3}$, there exists $x \in X \backslash R_{1}$ such that $g(x)=y_{1}$; hence.

$$
\pi_{Y_{1}}(F(x))=g(x)=y_{1} .
$$

Therefore, we have shown that $n \geq 1$. Now, suppose by way of contradiction that $n>1$. Then, there exist $a \neq b$ in $X$ such that

$$
\pi_{Y_{1}}(F(a))=y_{1}=\pi_{Y_{1}}(F(b)) .
$$

We obtain a contradiction in each of the following three cases (which take care of all posssibilities).

Case 1: $a, b \in R_{1}$. Then, $a=x_{\alpha}$ and $b=x_{\beta}$ with $\alpha \neq \beta$; hence,

$$
y_{\alpha}^{1}=\pi_{Y_{1}}(F(a))=\pi_{Y_{1}}(F(b))=y_{\beta}^{1} ;
$$

however, since $\alpha \neq \beta, y_{\alpha}^{1} \neq y_{\beta}^{1}$.

Case 2: $a, b \in X \backslash R_{1}$. Then,

$$
g(a)=\pi_{Y_{1}}(F(a))=\pi_{Y_{1}}(F(b))=g(b),
$$

which contradicts that $g$ is one-to-one.

Case 3: $a \in R_{1}, b \in X \backslash R_{1}$. Since $a \in R_{1}, a=x_{\alpha}$ for some $\alpha$, so

$$
\pi_{Y_{1}}(F(a))=y_{\alpha}^{1} \in R_{3} .
$$

Since $b \in X \backslash R_{1}$ and $g: X \backslash R_{1} \rightarrow Y_{1} \backslash R_{3}$,

$$
\pi_{Y_{1}}(F(b))=g(b) \in Y_{1} \backslash R_{3} .
$$

However, $(*)$ and $(\#)$ contradict that $\pi_{Y_{1}}(F(a))=\pi_{Y_{1}}(F(b))$.

This completes the proof that $F$ satisfies condition (i).

To show that $F$ satisfies condition (ii) of our lemma, let $C \in \mathcal{C}$. By Lemma 4.1, it suffices to show that $F$ intersects every closed separator of $C \times\left(Y_{1} \times Y_{2}\right)$. Let $T$ be a closed separator of $C \times\left(Y_{1} \times Y_{2}\right)$. We take two cases (which represent all possibilities by Lemma 5.1):

Case 1: $T$ is a type 1 separator of $C \times\left(Y_{1} \times Y_{2}\right)$. Then

$$
H_{T}=\left\{x \in C: \pi_{X}^{-1}(x) \subseteq T\right\} \text { is a separator of } C .
$$

Hence, $H_{T} \neq \emptyset$. Thus, $\exists p \in H_{T}$. Therefore, since any function $k: C \rightarrow Y_{1} \times Y_{2}$ intersects $\pi_{C}^{-1}(p), F \cap T \neq \emptyset$.

Case 2: $T$ is a type 2 separator of $C \times\left(Y_{1} \times Y_{2}\right)$. Then, $T=T_{\alpha}$ for some $\alpha$. Thus, since the sequences $R_{i}$ satisfy condition (1), $\left(x_{\alpha}, y_{\alpha}^{1}, y_{\alpha}^{2}\right) \in T$. Therefore, since $F\left(x_{\alpha}\right)=\left(y_{\alpha}^{1}, y_{\alpha}^{2}\right), F \cap T \neq \emptyset$. 
We state two corollaries to Lemma 6.1 we do not use the corollaries later, but we feel that the corollaries are of interest in themselves.

Corollary 6.1. If $Y$ is a nondegenerate $\mathfrak{c}$-connected separable metric space and $I \subseteq \mathbb{R}$ is a nondegenerate interval, then there is a connectivity bijection $g: I \rightarrow Y$ with dense graph.

Proof. Let $\mathcal{C}=\{C \subseteq I: C$ is connected and nondegenerate $\}$; notice that $|\mathcal{C}|=\mathfrak{c}$. Applying Lemma 6.1 with $Y_{1}=Y_{2}=Y$, there is a function $F: I \rightarrow\left(Y_{1} \times Y_{2}\right)$ such that

(i) $\left|\left\{x \in X: y_{i}=\pi_{Y_{i}}(F(x))\right\}\right|=1$ for every $y_{i} \in Y_{i}$ where $i \in\{1,2\}$ and

(ii) $F \mid C$ is connected and dense in $C \times\left(Y_{1} \times Y_{2}\right)$ for each $C \in \mathcal{C}$.

By (ii) and our choice of $\mathcal{C}, F$ is a connectivity function. Let $g=\pi_{Y_{1}} \circ F$. Since $g$ is the composition of a connectivity function followed by a continuous function, it follows from [4, Lemma 4.1] that $g$ is connectivity. By (i) we know that $g$ is a bijection. By (ii) $g$ is dense in $I \times Y$.

In connection with the corollary just proved note that the only connectivity bijections of $[0,1]$ into itself are homeomorphisms.

Corollary 6.2. If $Y$ is a nondegenerate $\mathfrak{c}$-connected separable metric space and $X$ is a nondegenerate continuum, then there is a bijection $g: X \rightarrow Y$ such that $g \mid C$ is connected and dense in $C \times Y$ for any nondegenerate subcontinuum $C$ of $X$.

Proof. The proof is similar to the proof of Corollary 6.1 with the modification that $\mathcal{C}=\{C \subseteq X: C$ is a nondegenerate subcontinuum of $X\}$.

\section{Theorem 7.1}

We state Theorem 7.1 and two corollaries, after which we prove Theorem 7.1

Theorem 7.1. Let $Y$ be a nondegenerate separable metric space. If $Y$ is a weak $S_{4}$ space, then $Y$ is not $\mathfrak{c}$-connected.

Corollary 7.1. If a nondegenerate continuum $X$ is a weak $S_{4}$ space, then $X$ can be separated by countably many points.

Proof. For complete separable metric spaces, having no countable separator and being c-connected are equivalent. This follows from [5, Theorem 3 p. 155] and the fact that uncountable closed subsets of complete separable metric spaces have cardinality $\mathfrak{c}$.

Corollary 7.2. If a nondegenerate continuum $X$ is a weak $S_{4}$ space, then $X$ is hereditarily decomposable.

Proof. If $X$ is indecomposable, then $X$ has uncountably many mutually disjoint composants, each of which is a dense connected subset of $X$ [8, pp. 83 and 204]; so, no countable set can separate $X$, and hence $X$ would not be weak $S_{4}$ by Corollary 7.1. Thus, no nondegenerate indecomposable continuum is a weak $S_{4}$ space. Since being weak $S_{4}$ is hereditary by Proposition 3.1, any weak $S_{4}$ continuum must be hereditarily decomposable.

We use the following two lemmas in the proof of Theorem 7.1 . 
Lemma 7.1. Let $Y_{1}$ and $Y_{2}$ be $\mathfrak{c}$-connected separable metric spaces. Then there is a dense connectivity function $h:[0,1) \rightarrow Y_{1} \times Y_{2}$ such that

$$
\left|\left\{x \in[0,1): y_{i}=\pi_{Y_{i}}(h(x))\right\}\right|=1
$$

for every $y_{i} \in Y_{i}$ where $i \in\{1,2\}$.

Proof. Let $\mathcal{C}=\{C \subseteq[0,1): C$ is connected and nondegenerate $\}$. Notice that $|\mathcal{C}|=$ $\mathfrak{c}$ and apply Lemma 6.1 .

In what follows, consider the unit circle $S^{1}$ as the disjoint union of

$$
A=\{(\cos (\theta), \sin (\theta)): \theta \in[0, \pi)\} \quad \text { and } B=\{(\cos (\theta), \sin (\theta)): \theta \in[\pi, 2 \pi)\} .
$$

Note that $A$ and $B$ are both homeomorphic to $[0,1)$. For each point $p \in S^{1}$, we let $-p$ denote the point antipodal to $p$. The homeomorphism of $S^{1}$ onto itself defined by $p \rightarrow-p$ is denoted by $\rho$. We will let $Y$ be a fixed nondegenrate $\mathfrak{c}$ connected separable metric space, and we let $Y=Y_{1} \cup Y_{2}$, where $Y_{1}$ and $Y_{2}$ are as in Lemma 4.2

Lemma 7.2. Identifying $[0,1)$ and $A$, let $F: A \rightarrow Y_{1} \times Y_{2}$ be the dense connectivity function guaranteed by Lemma 7.1. Then the function $F^{*}: S^{1} \rightarrow Y$ defined by

$$
F^{*}(x)= \begin{cases}\pi_{Y_{1}}(F(x)) & \text { if } x \in A ; \\ \pi_{Y_{2}}(F(-x)) & \text { if } x \in B\end{cases}
$$

is a bijection and for any connected subset $C$ of $S^{1}$, the set

$$
P(C)=\left\{\left\{\left(x, F^{*}(x)\right),\left(-x, F^{*}(-x)\right)\right\}: x \in C\right\}
$$

is a connected subset of the 2-fold symmetric product $F_{2}\left(S^{1} \times Y\right)$.

Proof. That $F^{*}$ is a bijection follows from (i) of Lemma 7.1 and the fact that $Y$ is the disjoint union of $Y_{1}$ and $Y_{2}$.

Let $C \subseteq S^{1}$ be connected. We prove $P(C)$ is connected by considering four cases:

Case 1: $C \subseteq A$.

Since $F$ is a connectivity function, $F \mid C$ is connected. Define $\Theta: F \mid C \rightarrow P(C)$ by $\Theta((x, F(x)))=\left\{\left(x, F^{*}(x)\right),\left(-x, F^{*}(-x)\right)\right\}$. The function $\Theta$ is easily checked to be continuous, so $P(C)=\Theta(F \mid C)$ is connected.

Case 2: $C \subseteq B$.

There is a $D \subseteq A$ such that $-C=D$. By Case $1, P(D)$ is connected. Notice that $P(D)=P(C)$, so $P(C)$ is connected.

Case 3: $C_{1}=C \cap A$ and $C_{2}=C \cap B$ are both connected and non-empty.

By the first two cases we know that $P\left(C_{1}\right)$ and $P\left(C_{2}\right)$ are both connected. Let $p \in \operatorname{cl}\left(C_{1}\right) \cap \operatorname{cl}\left(C_{2}\right)$. Assume $p \in C_{2}$. It is enough for us to show that $\left\{\left(p, F^{*}(p)\right),\left(-p, F^{*}(-p)\right)\right\} \in \operatorname{cl}\left(P\left(C_{1}\right)\right)$. Since $F \mid C_{1}$ is dense in $C_{1} \times\left(Y_{1} \times Y_{2}\right)$, it follows that there is a sequence of points $p_{n} \in C_{1}$ such that $\lim _{n \rightarrow \infty} p_{n}=p$ and $\lim _{n \rightarrow \infty} F\left(p_{n}\right)=F(p)$. In particular, we have $\lim _{n \rightarrow \infty} \pi_{Y_{1}}\left(F\left(p_{n}\right)\right)=\pi_{Y_{1}}(F(p))$ and $\lim _{n \rightarrow \infty} \pi_{Y_{2}}\left(F\left(p_{n}\right)\right)=\pi_{Y_{2}}(F(p))$. It follows that

$$
\lim _{n \rightarrow \infty}\left\{\left(p_{n}, F^{*}\left(p_{n}\right)\right),\left(-p_{n}, F^{*}\left(-p_{n}\right)\right)\right\}=\left\{\left(p, F^{*}(p)\right),\left(-p, F^{*}(-p)\right)\right\} .
$$

Thus, $\left\{\left(p, F^{*}(p)\right),\left(-p, F^{*}(-p)\right)\right\} \in \operatorname{cl}\left(P\left(C_{1}\right)\right)$ so $P(C)$ is connected. When $p \in C_{1}$, then we argue that $\left\{\left(p, F^{*}(p)\right),\left(-p, F^{*}(-p)\right)\right\} \in \operatorname{cl}\left(P\left(C_{2}\right)\right)$. Since $C_{2}=-D$ for some $D \subseteq A$ and $\rho$ is a homeomorphism, it follows that $(F \circ \rho) \mid C_{2}$ is dense in $C_{2} \times\left(Y_{1} \times Y_{2}\right)$; the argument now proceeds as in the case when $p \in C_{2}$. 
Case 4: $C_{1}=C \cap A$ and $C_{2}=C \cap B$ are non-empty but not both are connected.

It is easy to check that either $C_{1}=A$ or $C_{2}=B$. Assume that $C_{2}=B$; then $C_{1}$ is the union of two separated connected sets $G_{1}$ and $G_{2}$ such that $(-1,0) \in \operatorname{cl}\left(G_{1}\right)$ and $(1,0) \in G_{2}$. Arguing as in the Case 3 , we have that $P\left(C_{2} \cup G_{1}\right)$ and $P\left(C_{2} \cup G_{2}\right)$ are connected. Since $P\left(C_{2}\right)$ is connected by Case 2, it follows that $P(C)$ is connected. The case when $C_{1}=A$ is similar.

Proof of Theorem 7.1. Suppose, to the contrary, that there is a $\mathfrak{c}$-connected weak $S_{4}$ space $Y$. By Lemma 4.2, there are disjoint c-connected dense sets, $Y_{1}$ and $Y_{2}$, in $Y$ such that $Y=Y_{1} \cup Y_{2}$. Let

$$
F: A \rightarrow Y_{1} \times Y_{2} \quad, \quad F^{*}: S^{1} \rightarrow Y
$$

be as in Lemma 7.2.

Since $F^{*}: S^{1} \rightarrow Y$ is one-to-one (Lemma 7.2), $\pi_{Y} \mid F^{*}: F^{*} \rightarrow Y$ is one-to-one. Thus, by Proposition $3.2, F^{*}$ is a weak $S_{4}$ space. Therefore, letting

$$
Q=\left\{\left\{\left(x, F^{*}(x)\right),\left(-x, F^{*}(-x)\right)\right\}: x \in S^{1}\right\}
$$

there is a continuous selector $\Sigma: Q \rightarrow F^{*}$.

We use $\Sigma$ to obtain a contradiction. Let

$$
\begin{gathered}
V=\left\{(1, \theta) \in S^{1}:-\frac{\pi}{2}<\theta<\frac{\pi}{2}\right\}, \\
\Lambda=\left\{\left\{\left(x, F^{*}(x)\right),\left(-x, F^{*}(-x)\right)\right\}: x \in V\right\} .
\end{gathered}
$$

Since $\Lambda$ is the set $P(V)$ in Lemma 7.2, $\Lambda$ is connected. Thus, since $\Lambda \subseteq Q$ and $\Sigma: Q \rightarrow F^{*}$ is a continuous selector, we see that $\Sigma(\Lambda)$ is a connected subset of $\left(F^{*} \mid V\right) \cup\left(F^{*} \mid-V\right)$. Therefore, since $F^{*} \mid V$ and $F^{*} \mid-V$ are mutually separated, we see that $\Sigma(\Lambda) \subseteq F^{*} \mid V$ or $\Sigma(\Lambda) \subseteq F^{*} \mid-V$. Hence, without loss of generality, we can assume that

$$
\Sigma(\Lambda) \subseteq F^{*} \mid V .
$$

Now, let $W=\left\{(1, \theta) \in S^{1}: 0<\theta<\pi\right\}$. Let

$$
\Gamma=\left\{\left\{\left(x, F^{*}(x)\right),\left(-x, F^{*}(-x)\right)\right\}: x \in W\right\} .
$$

By the argument above, we see that

$$
\Sigma(\Gamma) \subseteq F^{*} \mid W \quad \text { or } \Sigma(\Gamma) \subseteq F^{*} \mid-W .
$$

Case 1: $\Sigma(\Gamma) \subseteq F^{*} \mid W$. Let $p=\left(1, \frac{3 \pi}{4}\right) \in S^{1}$ (note that $p \in W \cap(-V)$ ), and let

$$
D=\left\{\left(p, F^{*}(p)\right),\left(-p, F^{*}(-p)\right)\right\} .
$$

Since $D \in \Gamma$ and since $\Sigma(\Gamma) \subseteq F^{*}\left|W, \Sigma(D) \in F^{*}\right| W$. Thus, since $-p \notin W$, we see that

$$
\Sigma(D)=\left(p, F^{*}(p)\right) .
$$

Since $-p \in V$, we know that $D \in \Lambda$; thus, since $\Sigma(\Lambda) \subseteq F^{*}\left|V, \Sigma(D) \in F^{*}\right| V$. Therefore, since $p \notin V$, we see that

$$
\Sigma(D)=\left(-p, F^{*}(-p)\right) .
$$

By (1) and (2), we have a contradiction. 
Case 2: $\Sigma(\Gamma) \subseteq F^{*} \mid-W$. Let $q=\left(1, \frac{\pi}{4}\right) \in S^{1}$ (note that $\left.q \in W \cap V\right)$. Then, letting

$$
E=\left\{\left(q, F^{*}(q)\right),\left(-q, F^{*}(-q)\right)\right\}
$$

we can apply the ideas used in Case 1 to obtain a contradiction.

Since Case 1 and Case 2 each led to a contradiction, we have a contradiction to our supposition at the beginning of the proof of the theorem.

\section{REFERENCES}

[1] R. G. Gibson and T. Natkaniec, Darboux-like functions, Real Anal. Exchange 22(2) (199697), 492-533. MR 98h:26004

[2] M. Hagan, Equivalence of connectivity maps and peripherally continuous transformations, Proc. Amer. Math. Soc. 17 (1966), 175-177. MR 33:3268

[3] O. H. Hamilton, Fixed points for certain noncontinuous transformations, Proc. Amer. Math. Soc. 8 (1957), 750-756. MR 19:301b

[4] J. Jastrzȩbski, J. Jẹdrzejewski, and T. Natkaniec, On some subclasses of Darboux functions, Fund. Math. 138 (1991), 165-173. MR 92h:26006

[5] K. Kuratowski, Topology, Vol. 2, Academic Press and Polish Scientific Publishers, 1968. MR 41:4467

[6] E. Michael, Topologies on spaces of subsets, Trans. Amer. Math. Soc. 71(1951), 152-182. MR 13:54f

[7] S. B. Nadler, Jr., Hyperspaces of sets, Marcel Dekker Inc., New York, Basel 1978. MR 58:18330

[8] S. B. Nadler, Jr., Continuum Theory, Marcel Dekker Inc., New York and Basel and Hong Kong 1992. MR 93m:54002

Department of Mathematics, University of Louisville, Louisville, Kentucky 402920001

Department of Mathematics, West Virginia University, Morgantown, West Virginia 26506-6310 